\title{
Publisher Correction: Memristive crossbar arrays for brain-inspired computing
}

Qiangfei Xia (D) and J. Joshua Yang (D)

Correction to: Nature Materials https://doi.org/10.1038/s41563-019-0291-x, published online 20 March 2019.

In the version of this Review originally published, an older version of Fig. 3 was used by mistake. Panels $\mathbf{a}, \mathbf{b}$ and $\mathbf{f}$ of the old figure have now been removed, and the panels have been relabelled sequentially. This does not affect any of the text. Also, the scale bar in the first image in Table 1 was incorrectly labelled $50 \mu \mathrm{m}$; it should have been $500 \mu \mathrm{m}$. 